\title{
Free convection from an arbitrarily inclined plate in a porous medium
}

\author{
I. Pop, T.-Y. Na
}

\begin{abstract}
A mathematical model for the flow and heat transfer in the free convection from an arbitrary inclined isothermal flat plate embedded in a porous medium is presented, in which the Darcy-Boussinesq approximation is adopted to account for bouyancy force. A novel inclination parameter $\xi$ is proposed such that all cases of the horizontal, inclined and vertical plates can be described by a single set of transformed boundary layer equations. Moreover, the similarity equations for the limiting cases of the horizontal and vertical plates are recovered from the transformed equations by setting $\xi=0$ and $\xi=1$, respectively. Detailed results for the skin friction coefficient and Nusselt number as well as for the dimensionless velocity and temperature profiles are presented for a wide range of the parameter $\xi$. A comparison with similarity solution shows excellent agreement.
\end{abstract}

Freie Konvektion an einer beliebig geneigten Platte in einem porösen Medium

Zusammenfassung Es wird ein mathematisches Modell zur Beschreibung des Strömungsverhaltens und des Wärmeübergangs bei freier Konvektion an einer beliebig geneigten, isothermen, ebenen Platte, die in ein poröses Medium eingebettet ist, vorgestellt, wobei die Auftriebskraft über die Darcy/Boussinesq-Approximation erfaßt wird. Durch die Einführung eines Neigungsparameters $\zeta$ lassen sich zwischen vertikal und horizontal beliebig orientierte Platten durch einen einzigen Satz transformierter Grenzschichtgleichungen beschreiben. Für die Grenzfälle $\zeta=0$ (horizontal) und $\zeta=1$ (vertikal) liefern die transformierten Gleichungen die bekannten Ähnlichkeitsbeziehungen. Für einen weiten Bereich des Parameters $\zeta$ werden Ergebnisse bezüglich Reibungsbeiwert

Received 1 February 1996

\section{Pop}

Faculty of Mathematics

University of Cluj

R-3400 Cluj, CP253

Romania

Tsung-Yen $\mathrm{Na}$

Department of Mechanical Engineering

University of Michigan - Dearborn

Dearborn

MI, 48128

USA

Correspondence to: I. Pop und Nusselt-Zahl, so wie die dimensionslosen Geschwindigkeitsund Temperaturprofile mitgeteilt. Beim Vergleich mit Ähnlichkeitslösungen zeigt sich ausgezeichnete Übereinstimmung.

\section{Nomenclature}

$C_{f} \quad$ skin friction coefficient defined in Eq. (17)

$f \quad$ reduced stream function defined in Eq. (8)

$g \quad$ acceleration due to gravity

$K \quad$ permeability of the porous medium

$\mathrm{Nu} \quad$ Nusselt number defined in Eq. (17)

$q_{w} \quad$ heat flux from the plate

$\mathrm{Ra} \quad$ Rayleigh number

$T \quad$ fluid temperature

$T_{w} \quad$ wall temperature

$T_{\infty} \quad$ temperature of ambient fluid

$u, v \quad$ velocity components along $(x, y)$-axes

$U_{c} \quad$ characteristic velocity

$x, y \quad$ Cartesian coordinates along the normal to the plate, respectively

\section{Greek symbols}

\section{$\alpha \quad$ thermal diffusivity}

thermal expansion coefficient

inclination parameter defined in Eq. (5)

parameter defined in Eq. (7)

pseudo-similarity variable defined in Eq. (6)

nondimensional temperature defined in Eq. (8)

thermal conductivity

dynamic viscosity

kinematic viscosity

variable defined in Eq. (6)

density

wall skin friction

angle of inclination measured from the horizontal stream function

Superscript

differentiation with respect to $\eta$

\section{Introduction}

A classical thermal convection in porous media which is relevant to the study of geothermal activities, underground transport of pollutants, paper processing, building insulation, drying of grains, and solar pond design is that of heating from horizontal, vertical and inclined surfaces. Free convection from horizontal and vertical surfaces embedded in a porous medium 
has been investigated in great details both theoretically and experimentally. An account of most of the work carried out for these problems before 1992 can be found in the book by Nield and Bejan [1]. However, free convection along inclined surfaces has received less attention than the cases of vertical and horizontal plates. Rees and Riley [2], Ingham et al. [3], and Kumari et al. [4] have presented detailed analytical and numerical solutions to the problem of free convection along a flat plate in a porous medium which are valid only for inclined plates at small angles to the horizontal. These solutions are, however, not valid uniformly from the horizontal limit to the vertical limit, respectively.

The purpose of this paper is to study free convection above an inclined isothermal flat plate embedded in a porous medium by introducing new coordinates which permit rigorous solutions for the entire range of inclination from the horizontal case to the vertical case, respectively. Consequently, a set of two coupled non-similar equations is obtained which is solved numerically using a very efficient finite difference method proposed by Keller [6]. Accurate numerical solutions are obtained for both the cases of positively inclined plate $\left(0^{\circ} \leq \phi \leq 90^{\circ}\right)$ and negatively inclined plate at small angles to the horizontal $\left(\phi \leq 0^{\circ}\right)$. Of interest are the effect of inclination plate parameter on the velocity and temperature profiles as well as on the skin friction coefficient and Nusselt number. The known results for the classical situations of horizontal and vertical plates in a porous medium first studied by Cheng and Chang [6] and, respectively, by Cheng and Minkowycz [7] were recovered.

It may be noted to this end that important contributions to the problem of free convection from arbitrarily inclined plate in a viscous (non-porous) fluid were made by Yu and Liu [8], Lin et al. [9], and Umemura and Law [10].

\section{2}

\section{Basic equations}

Consider the steady boundary layer free convection flow above a semi-infinite flat plate which is inclined at an angle $\phi$ to the horizontal and embedded in a fluid-saturated porous medium. The inclination angle is either positive $\left(0^{\circ} \leq \phi \leq 90^{\circ}\right)$ or slightly negative $\left(\phi \leq 0^{\circ}\right)$. The plate is maintained at a uniform temperature $T_{w}$, while the quiescent ambient fluid is at the lower temperature $T_{\infty}$. The physical model and coordinate system is shown schematically in Fig. 1. On the basis of Darcy-Boussinesq approximation, the velocity and temperature within the momentum and thermal boundary layers which develop along the inclined plate are governed by the following equations:

$$
\begin{aligned}
& \frac{\partial u}{\partial x}+\frac{\partial v}{\partial y}=0 \\
& \frac{\partial u}{\partial y}=\frac{g K \beta}{v}\left(\frac{\partial T}{\partial y} \sin \phi-\frac{\partial T}{\partial x} \cos \phi\right) \\
& u \frac{\partial T}{\partial x}+v \frac{\partial T}{\partial y}=\alpha \frac{\partial^{2} T}{\partial y^{2}}
\end{aligned}
$$

which are to be solved subject to the boundary conditions $v=0, T=T_{w} \quad$ on $y=0 ; \quad u=0, T=T_{\infty} \quad$ as $y \rightarrow \infty$
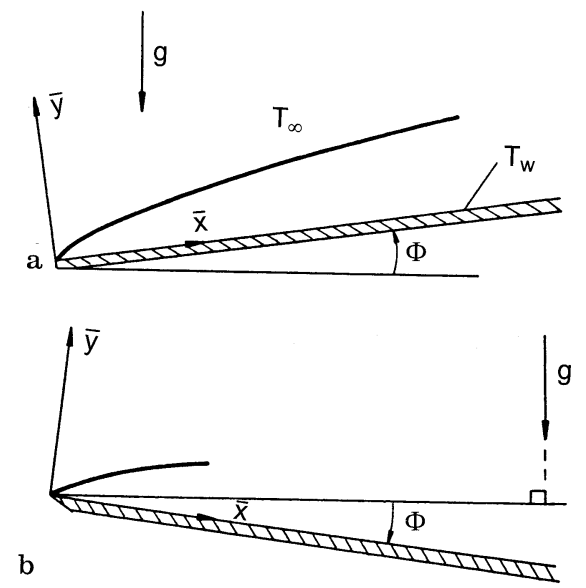

Fig. 1a,b. Physical model and coordinate system

Here $(x, y)$ are Cartesian coordinates along and normal to the plate, $(u, v)$ are the velocity components along $(x, y)$-axes, $T$ is the temperature, $g$ is the acceleration due to gravity, $K$ is the porosity, $v, \beta$ and $\alpha$ are the kinematic viscosity, coefficient of thermal expansion and thermal diffusivity, respectively.

To transform Eqs. (1)-(3) which describe free convection flow from an arbitrarily inclined plate in a porous medium, we introduce a nondimensional parameter of the form

$\zeta=\frac{(R a|\sin \phi|)^{1 / 2}}{(R a \cos \phi)^{1 / 3}}$

where $R a=g K \beta\left(T_{w}-T_{\infty}\right) x / \alpha v$ is the Rayleigh number. This parameter is the porous medium analogue of the one proposed by $\mathrm{Yu}$ and Liu [8] in a viscous fluid. It describes the relative strength of the longitudinal to the normal components of the buoyancy force acting simultaneously on the boundary layer. It also serves as a stretched longitudinal coordinate for a fixed inclined angle. Further, we introduce the following variables

$\xi(x)=\frac{\zeta}{(1+\zeta)}, \quad \eta=\left(\frac{y}{x}\right) \lambda$

where

$\lambda=(R a \cos \phi)^{1 / 3}+(R a|\sin \phi|)^{1 / 2}$

It is worth mentioning that the coordinate $\xi$ measures the downstream distance along the plate from the leading edge for a specified inclination angle. It also represents a parameter of inclination since it varies from $\xi=0$ to $\xi=1$ when the inclined angle varies from $\phi=0^{\circ}$ to $\phi=90^{\circ}$ for a fixed Rayleigh number.

In addition to the variables (6), we introduce the reduced stream function $f$ and dimensionless temperature $\theta$ according to

$f(\xi, \eta)=\frac{\psi}{\alpha \lambda}, \quad \theta(\xi, \eta)=\frac{T-T_{\infty}}{T_{w}-T_{\infty}}$

where $\psi$ is the stream function which is defined as

$u=\frac{\partial \psi}{\partial y}, \quad v=-\frac{\partial \psi}{\partial x}$ 
Hence Eqs. (1) to (3) can be transformed as

$f^{\prime \prime}-\frac{1}{6}(4-\xi)(1-\xi)^{3} \eta \theta^{\prime}= \pm \xi^{2} \theta^{\prime}-\frac{1}{6} \xi(1-\xi)^{4} \frac{\partial \theta}{\partial \xi}$

$\theta^{\prime \prime}+\frac{1}{6}(2+\xi) f \theta^{\prime}=\xi(1-\xi)\left(f^{\prime} \frac{\partial \theta}{\partial \xi}-\theta^{\prime} \frac{\partial f}{\partial \xi}\right)$

subject to the boundary conditions

$f=0, \theta=1, \quad$ on $\eta=0, \quad f^{\prime}=0, \theta=0 \quad$ as $\eta \rightarrow \infty$

where primes denote differentiation with respect to $\eta$. Equations (10) and (11) are the universal formulation for free convection from an isothermal plate of arbitrary inclinations.

The plus and minus signs before the first term in the righthand side of Eq. (10) pertain to the positive and negative inclination cases, respectively. On the other hand, we notice that Eqs. (10) and (11) are readily reduced to the following similarity equations for the limiting case of a horizontal plate $\left(\phi=0^{\circ}\right.$ and $\left.\xi=0\right)$ in a porous medium found by Cheng and Chang [6]

$f^{\prime \prime}-\frac{2}{3} \eta \theta^{\prime}=0$

$\theta^{\prime \prime}+\frac{1}{3} f \theta^{\prime}=0$

For the other limiting case of a vertical plate $\left(\phi=90^{\circ}\right.$ and $\left.\xi=1\right)$ embedded in a porous medium, Eqs. (10) and (11) reduce to

$f^{\prime \prime}=\theta^{\prime}$

$\theta^{\prime \prime}+\frac{1}{2} f \theta^{\prime}=0$

first obtained by Cheng and Minkowycz [7].

The physical quantities of interest are the skin friction coefficient and the Nusselt number which are defined as

$C_{f}=\frac{\tau_{w}}{\rho U_{c}^{2}}, \quad N u=\frac{x q_{w}}{\kappa\left(T_{w}-T_{\infty}\right)}$

where $U_{c}=\left(\alpha v / x^{2}\right)^{1 / 2}$ is the characteristic velocity, $\tau_{w}$ is the skin friction and $q_{w}$ is the heat flux at the wall which are given by

$\tau_{w}=\mu\left(\frac{\partial u}{\partial y}\right)_{y=0}, \quad q_{w}=-\kappa\left(\frac{\partial T}{\partial y}\right)_{y=0}$

Using (6) and (8), we obtain

$\frac{C_{f}}{(R a \cos \phi)}=(1+\zeta)^{3} f^{\prime \prime}(\xi, 0)$,

$\frac{N u}{(R a \cos \phi)^{1 / 3}}=(1+\zeta)\left[-\theta^{\prime}(\xi, 0)\right]$

\section{3}

\section{Results and discussions}

The two coupled partial differential equations (10) and (11) subject to the boundary conditions (12) were solved numerically by means of a very efficient finite-difference method proposed by Keller [5]. Since $\xi \sim 1 /\left(1+\operatorname{constant} \cdot x^{-1 / 6}\right)$, the numerical integration procedure has to be started at $\xi=0$ and marched forward step-by-step to $\xi=1$. To initiate the numerical process, the solution along $\xi=0$ is required, which is the similarity solution of free convection above a heated isothermal horizontal flat plate in a porous medium given by
Eqs. (13) and (14). The details of the numerical procedure can be found in Refs. [11-16].

\section{1}

\section{Positively inclined plate}

The numerical results for the skin friction coefficient and Nusselt number as a function of $\xi$ are shown in Figs. 2 and 3. The similarity solutions given by the set of equations $(13,14)$ at $\xi=0$ (horizontal plate) and $(15,16)$ at $\xi=1$ (vertical plate) are also shown in these figures for comparison. We notice that the agreement between the numerical solutions of the full boundary layer equations $(10,11)$ and the similarity solutions at $\xi=0$ and $\xi=1$ is excellent. Further, Fig. 2 shows that the skin friction coefficient increases monotonically as the plate is tilted from the horizontal $(\xi=0)$ toward the vertical $(\xi=1)$.

This is because the bouyancy force becomes stronger as $\xi$ increases from $\xi=0$ to $\xi=1$. On the other hand, the Nusselt number decreases from 0.422 at $\xi=0$ to 0.236 at $\xi \simeq 0.55$ and then increases to 0.444 at $\xi=1$, as can be seen in Fig. 3 .

Therefore, the Nusselt number exhibits the existence of a global minimum at $\xi \simeq 0.55(\zeta \simeq 1.22)$ where the tangential and normal components of buoyance force are comparable.

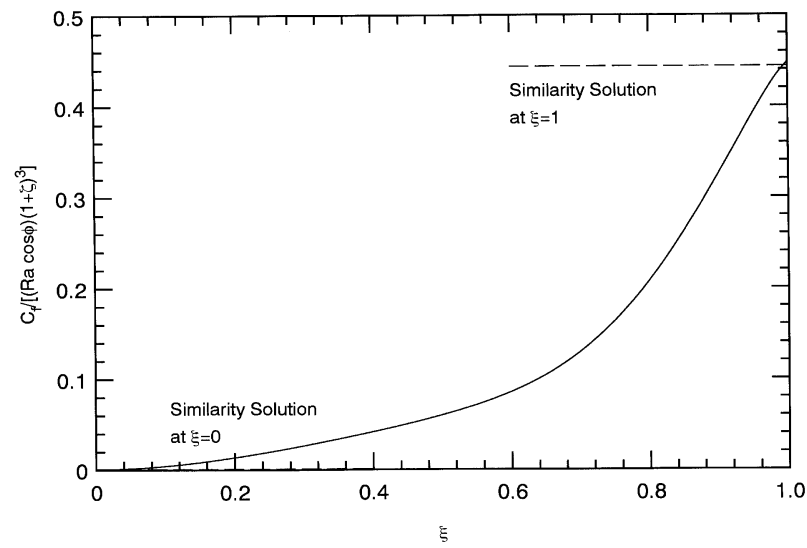

Fig. 2. Variation of the skin friction coefficient with $\xi$ for positive inclination

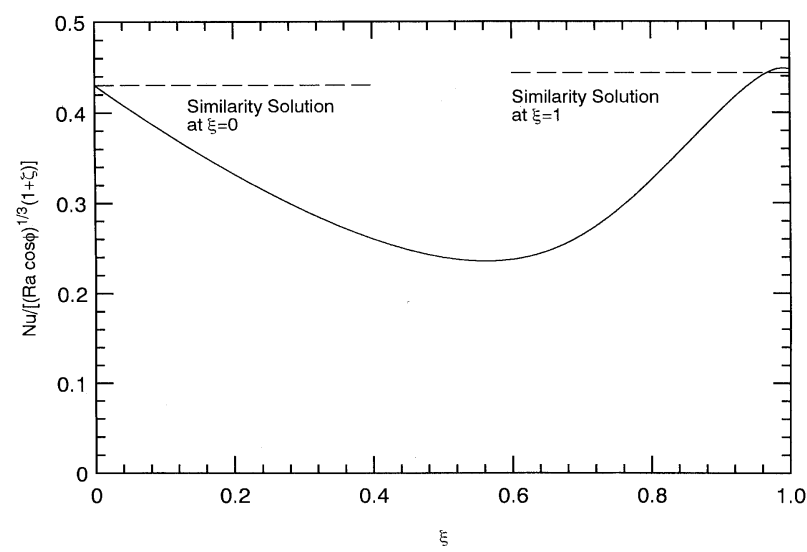

Fig. 3. Variation of the Nusselt number with $\xi$ for positive inclination 
The dimensionless velocity and temperature profiles are presented in Figs. 4 and 5 for $\xi=0,0.5$ and 1 . The evolution of the profiles from the horizontal limit to the vertical limit can be seen from these figures.

\section{2}

\section{Negatively inclined plate}

Figures 6 and 7 illustrate the variation of the skin friction coefficient and Nusselt number for negative inclination angles. The similarity solutions at $\xi=0$ are also shown in these figures. It is seen that both the skin friction coefficient and Nusselt number vanish at $\xi=\xi_{s} \sim 0.75$ when the plate is at a small negative angle to the horizontal. Therefore, the boundary layer separates downstream from the leading edge at $\xi_{s} \sim 0.75$. This trend is to be expected physically because the buoyancy force retards the flow motion when the plate is negatively inclined. A reverse flow region is expected downstream past the separation point beyond which the present solution procedure has to be terminated since the boundary layer approximation is no longer appropriate. However, the solution behaves in a regular manner at the separation point $\xi_{s} \sim 0.75$, and this is confirmed by the previous work [2-4].

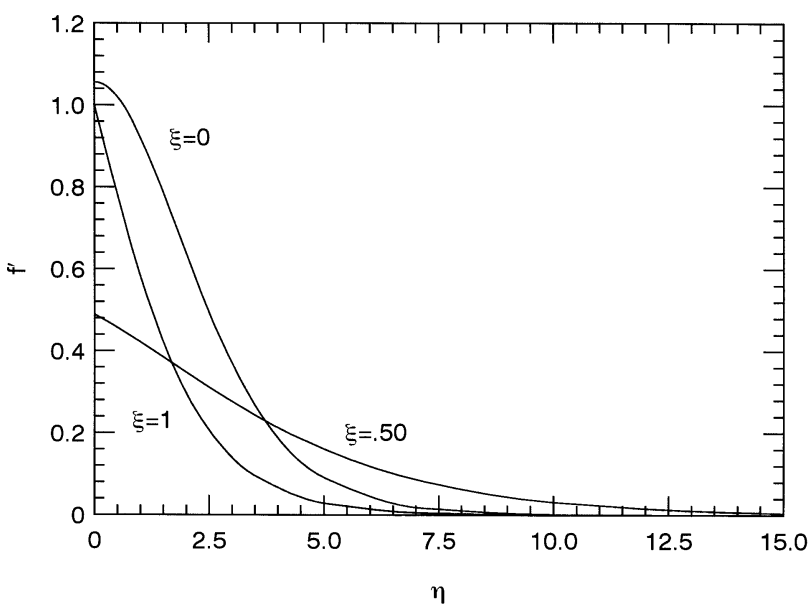

Fig. 4. Dimensionless velocity profiles for positive inclination

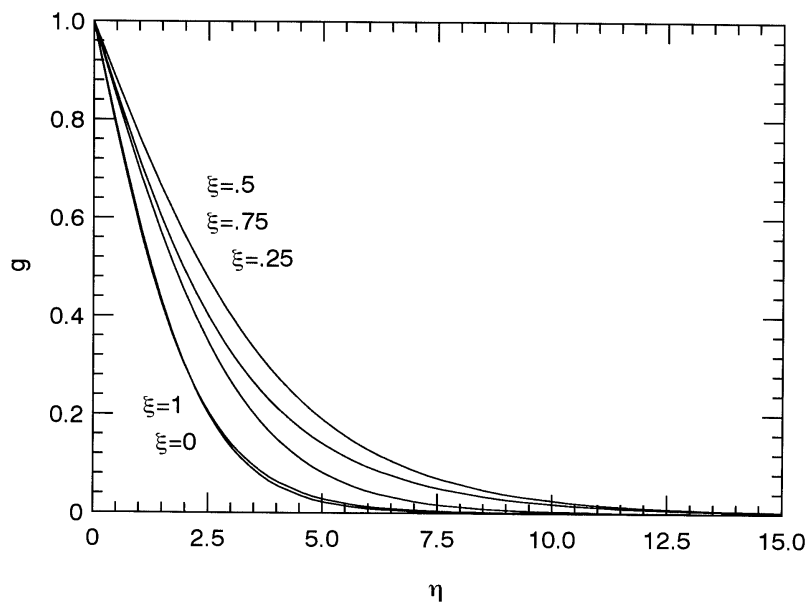

Fig. 5. Dimensionless temperature profiles for positive inclination
To this end, the dimensionless velocity and temperature profiles for negative inclination angles are depicted in Figs. 8 and 9 at $\xi=0,0.25$ and 0.5 . Note that the velocity profiles are steeper for lower values of $\xi$, while the temperature profiles

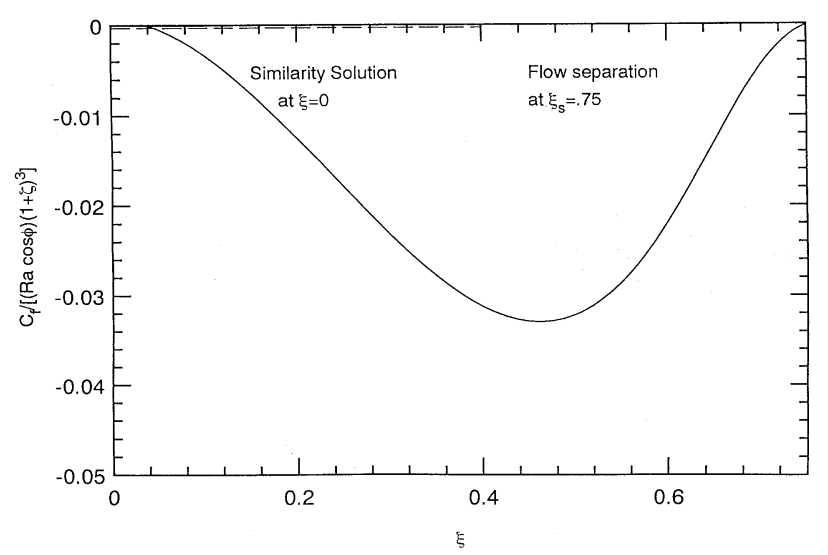

Fig. 6. Variation of the skin friction coefficient with $\xi$ for negative inclination

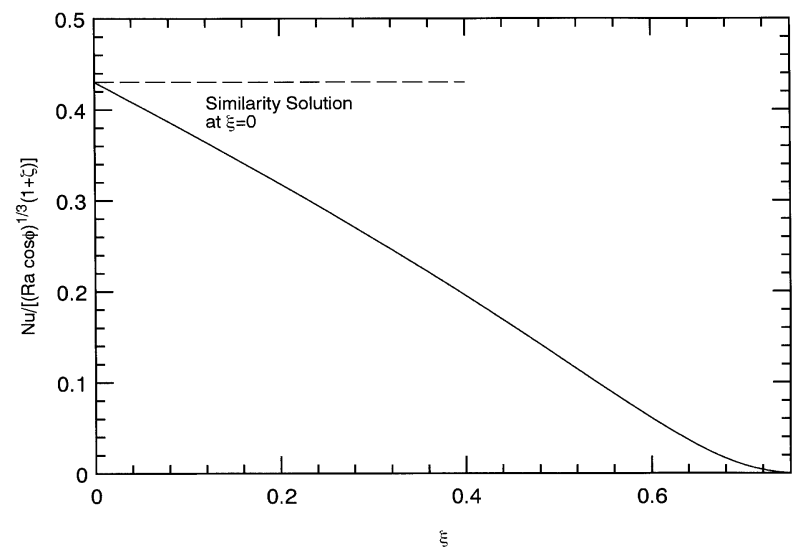

Fig. 7. Variation of the Nusselt number with $\xi$ for negative inclination

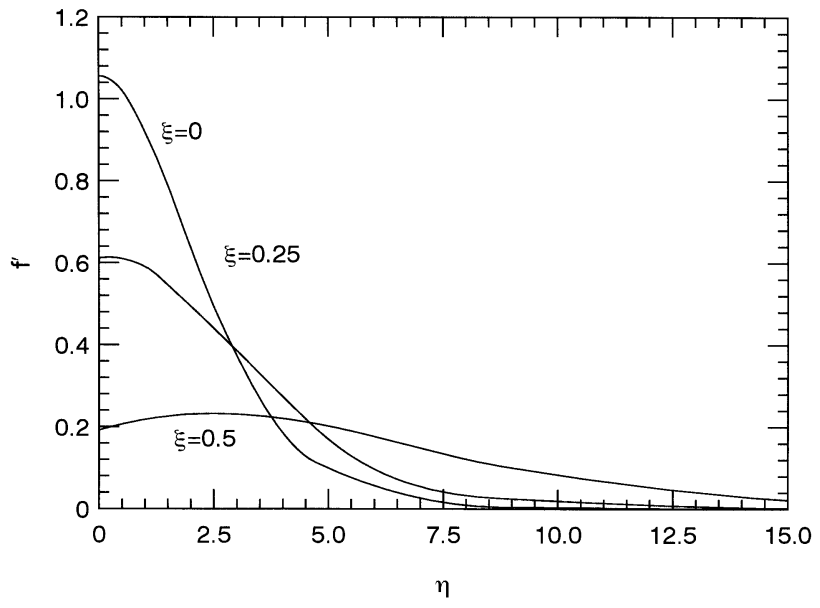

Fig. 8. Dimensionless velocity profiles for negative inclination 


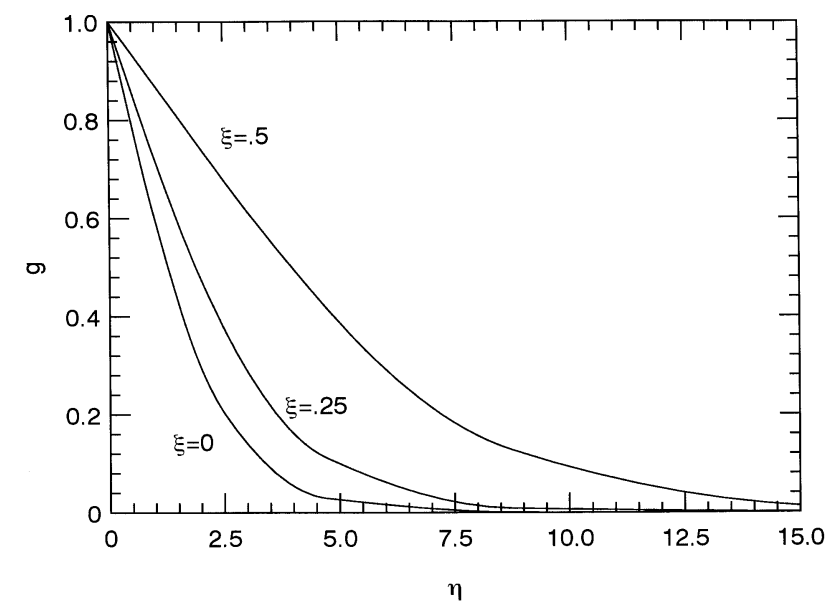

Fig. 9. Dimensionless temperature profiles for negative inclination

increase monotinically with increasing $\xi$. However, in the region just away from the plate, the velocity boundary layer thickness is larger for $\xi=0.5$ than for $\xi=0.25$ and $\xi=0$. This is to be expected due to the separation of the boundary layer at $\xi_{s} \sim 0.75$. It is also seen from Figs. 5 and 9 that the temperature profiles are flatter for negative inclination angles than for positive ones.

\section{4}

\section{Conclusions}

A novel formulation based on a proper inclination parameter and new coordinates has been proposed for the analysis of free convection above an isothermal inclined plate embedded in a porous medium. The attention was focused on both cases of positively inclined plate $\left(0^{\circ} \leq \phi \leq 90^{\circ}\right)$ and negatively inclined plate $\left(\phi \leq 0^{\circ}\right)$ at small angles to the horizontal. The resulting skin friction coefficient and Nusselt number as well as the dimensionless velocity and temperature profiles are determined numerically using the Keller box scheme. For the case of positive inclination it was found that the Nusselt number exhibits a global minimum at $\xi \sim 0.55(\zeta \sim 1.22)$ where the tangential and normal components of buoyancy force are comparable. It was also shown that for the case of negative inclination angles, the boundary layer separates downstream from the leading edge. We were able to determine very accurately the position of separation point at $\xi_{s} \sim 0.75$.
Finally, it is worth mentioning that our calculations agree excellently with the similarity solutions at $\xi=0$ (horizontal plate) and at $\xi=1$ (vertical plate) of Cheng and Chang [6], and Cheng and Minkowycz [7], respectively.

\section{References}

1. Nield, D.A.; Bejan, A.: Convection in Porous Media. Springer, Berlin, 1992

2. Rees, D.A.S.; Riley, D.S.: Free convection above a near horizontal semi-infinite heated surface embedded in a saturated porous medium. Int. J. Heat Mass Transfer 28 (1985) 183-190

3. Ingham, D.B.; Merkin, J.H.; Pop, I.: Natural convection from a semi-infinite flat plate inclined at a small angle to the horizontal in a saturated porous medium. Acta Mechanica 57 (1985) 185-202

4. Kumari, M.; Pop, I.; Nath, G.: Natural convection in porous media above a near horizontal uniform heat flux surface. Wärme- und Stoffübertr. 25 (1990) 155-159

5. Keller, H.B.: Numerical methods in boundary layer theory. Ann. Rev. Fluid Mech. 10 (1978) 417-433

6. Cheng, P.; Chang, I.D.: Bouyancy induced flows in a saturated porous medium adjacent to impermeable horizontal surfaces. Int. J. Heat Mass Transfer 11 (1976) 1267-1272

7. Cheng, P.; Minkowycz, W.J.: Free convection about a vertical flat plate embedded in a porous medium with application to heat transfer from a dike. J. Geophys. Res. 82 (1977) 2040-2044

8. Yu, W.-S.; Lin, H.-T.: Free convection heat transfer from an isothermal plate with arbitrary inclination. Wärme- und Stoffübertr. 23 (1988) 203-211

9. Lin, H.-T.; Yu, W.-S.; Yang, S.-L.: Free convection on an arbitrarily inclined plate with uniform surface heat flux. Wärmeund Stoffübertr. 24 (1989) 183-190

10. Umemura, A.; Law, C.K.: Natural-convection boundary layer flow over a heated plate with arbitrary inclination. J. Fluid Mech. 219 (1990) 571-584

11. Na, T.Y.: Numerical solution of natural convection flows past a non-isothermal vertical flat plate. Appl. Sci. Res. 33 (1978) 519-543

12. Na, T.Y.; Pop, I.: Free convection flow past a vertical flat plate embedded in a saturated porous medium. Int. J. Engng. Sci. 21 (1983) 517-526

13. Aziz, A.; Na, T.Y.: Perturbation Methods in Heat Transfer. Hemisphere, Washington, 1984

14. Pop, I.; Na, T.Y.: Natural convection of a Darcian fluid about a wavy cone. Int. Comm. Heat Mass Transfer 21 (1994) 891-899

15. Pop, I.; Na, T.Y.: Natural convection from a wavy cone. Appl. Sci. Res. 54 (1995) 125-136

16. Na, T.Y.; Pop, I.: Flow and heat transfer over a longitudinal circular cylinder moving in parallel or reversely to a free stream. Acta Mechanica, Vol. 118, no. 1-4, October, 1996 